\title{
Integrated Starter Alternator Sizing for Micro / Mild Hybrid Vehicle Using Monte Carlo Simulation
}

\author{
Saikat Subhra Ghosh, Timothy J. Flack and Teng Long \\ Department of Engineering, University of Cambridge United Kingdom, Cambridge CB3 0FA \\ Email:ssg39@cam.ac.uk
}

\begin{abstract}
The Integrated Starter Alternator (ISA) needs to cater for a very wide range of torque and speed, in both motor and generator modes and in different functionalities, such as engine starting, alternator, regenerative braking and torque boost. Furthermore, each of these functionalities, being dependent on the drive cycle, means that determining the optimal ISA torque-speed characteristics is a challenging problem. In this paper, a Monte-Carlo simulation has been reported to find the most frequently operated regions in the torque-speed plane of an ISA, using stochastic drive cycles. This has been used to determine the optimal torque-speed characteristic to inform the ISA design specification. In turn, this gives a more robust coverage of different driving patterns compared to a conventional driving cycle-based design
\end{abstract}

\section{INTRODUCTION}

The ISA [1] of a micro / mild hybrid is used in the different modes of operation, such as high torque, low speed for engine cranking, and high-speed low torque for alternator mode when cruising. Further, the intermediate range of torque and speed will allow other hybrid functionality such as regenerative braking and torque boost. Such a wide range of operation, both in torque and speed makes the sizing of the electrical drive for the ISA [2] a challenging problem. To obtain the required torque-speed characteristics of a machine, it is necessary to have knowledge about the operating points of the machine (in the torque speed plane), and how frequently these points are incurred. IEC 60034 [3] defines several duty-cycles for electrical machines, starting from $\mathrm{S} 1$ to $\mathrm{S} 8$, from continuous to intermittent, which represents industrial drives operating scenario. However, for an ISA the duty cycle will be dependent on the driving scenario, which varies drastically. In general, this is a common problem for component sizing in the automobile industry, hence several drive cycles are adopted as well as for the different regulatory tests. Although drive cycles represent real-life driving scenarios, in practice it cannot be assumed that the actual driving profile will be similar to them. Hence an ISA torque-speed (T-S) characteristic developed based on drive cycle might not ensure performance and optimal design. Monte Carlo simulation [4] has been used for such scenarios in many applications where the actual operating conditions are difficult to model, but follow a statistical pattern. In this paper, a Monte Carlo simulation has been used to emulate many possible and realistic driving conditions. The T-S operating points of the ISA are collected for each of them, at the 1s interval. Finally, they have been plotted, to understand what the required T$\mathrm{S}$ operating points of the machine are, and how frequently they are used. This gives a better understanding to develop the machine design specification. The required drive torquespeed characteristics have been obtained by covering the most frequently used zones in the frequency plot. Further, less frequently operated conditions, which demand more torque or power, can be catered as peak operation, while the machine can be optimally sized to cater for the more frequently operated internal zone of the T-S characteristics on a continuous basis.

\section{Methodology}

For component sizing of automobiles, as well as the regulatory requirement, several driving cycles are used to emulate real-life driving scenario. In Fig 1(a) the speed-time profile of the New European Drive Cycle (NEDC) is shown. For any speed-time profile of the vehicle, the required traction force on the wheel can be calculated using the Road-load equation, as given in Eqn. 1.

$$
F=\mu_{r} m g \cos \theta+m g \sin \theta+\frac{1}{2} \rho A C_{d}\left(v-v_{a}\right)^{2}+m a
$$

Where the terms are described as below:

$m$ : the mass of the vehicle, $g$ : gravitational acceleration, $\theta$ : the slope of the road, $\rho$ : the density of air, $A$ : Frontal area of the vehicle, $C_{d}$ : Coefficient of drag resistance, $v$ : Velocity of the vehicle, $v_{a i r}$ : Velocity of air, and $a$ : Acceleration. Using wheel diameter, the torque and speed at the wheel can be obtained by the following equations:

$$
\begin{aligned}
\omega_{w h} & =\frac{v}{\pi D_{w h}} \\
T_{w h} & =\frac{F D_{w h}}{2}
\end{aligned}
$$

Where $D_{w h}$ is the wheel diameter (with inflated tire), $\omega_{w h}$ and $T_{w h}$ are the speed and torque at wheel shaft respectively. Further, with the knowledge of gear ratio, (including final drive/differential ratio) the torque and speed requirement at the engine shaft can be obtained. The details of the vehicle used for this simulation study is reported in table I

\section{A. Torque Speed requirement of ISA}

The torque-speed requirement at engine shaft, along with braking torque would have been sufficient for sizing calculation of an electric vehicle. However, in a micro / mild hybrid vehicle, the ISA supplies or regenerates only a fraction of this 
TABLE I

Simulated Vehicle Details

\begin{tabular}{|c|c|c|}
\hline Parameter & Value & Unit \\
\hline \hline Vehicle Kerb Weight & 1500 & $\mathrm{~kg}$ \\
\hline Vehicle net weight & 2000 & $\mathrm{~kg}$ \\
\hline Rolling Friction & 0.0105 & \\
\hline Drag Coefficient & 0.6 & \\
\hline Air density & 1.18 & $\mathrm{~kg} / \mathrm{m}^{3}$ \\
\hline Tire radius & 0.268 & \\
\hline Differential ratio & 3.31 & \\
\hline Front area & 2.1 & $\mathrm{~m}^{2}$ \\
\hline Gear Box & 8-speed ZF 8HP45 & \\
\hline
\end{tabular}

required or available torque. Hence an algorithm for torquespeed requirement at the machine shaft need to be considered. The algorithm used is as follows:

- The motor operation is permitted if battery State of Charge (SOC) $>30 \%$

- Alternator operation permitted if battery SOC $<90 \%$

- The ISA regenerates the braking torque, ( limited by its maximum torque) available at that speed

- The ISA starts the engine when shaft speed is below 500 $\mathrm{rpm}$, with a starting torque of $40 \mathrm{Nm}(120 \mathrm{Nm}$ at the engine shaft)

- The ISA provides total torque if it is less than $40 \mathrm{Nm}$ (to improve Fuel economy, cutting engine off at low torque) at engine shaft from speed 500-2000 RPM

- The ISA provides total torque if it is less than $20 \mathrm{Nm}$ (to improve Fuel economy, cutting engine off at low torque) at engine shaft from speed 2000-3000 RPM

- The ISA provides total torque if it is less than $10 \mathrm{Nm}$ (to improve Fuel economy, cutting engine off at low torque) at engine shaft from speed 3000-4000 RPM

- The ISA provides the torque boost if the torque required is more than red-line torque (maximum engine torque available) to provide extra torque (limited by its maximum torque capability)

- The ISA provides necessary extra torque (limited by its maximum torque at that speed) to keep the engine operating at the optimal efficiency line if the required torque is more than the baseline.

The ISA - Engine shaft gear ratio is taken to be 3. Further, as the ISA operation algorithm is dependent on the engine optimal efficiency line, the BSFC map of Saturn 1.91 engine from the ADVISOR database [?], has been used for this paper. Fig 1(b) shows the used BSFC map along with the maximum torque line and the base-line of operation.

\section{B. Monte Carlo Simulation}

As discussed earlier, standard driving cycles are the representative driving scenario, however, the real driving scenario can vary significantly from them. Hence to emulate those condition we have done a Monte Carlo simulation to find torque speed operating points of the ISA in the different possible driving scenario, and their frequency of occurrence. To do so, for each run, a drive cycle is to be generated
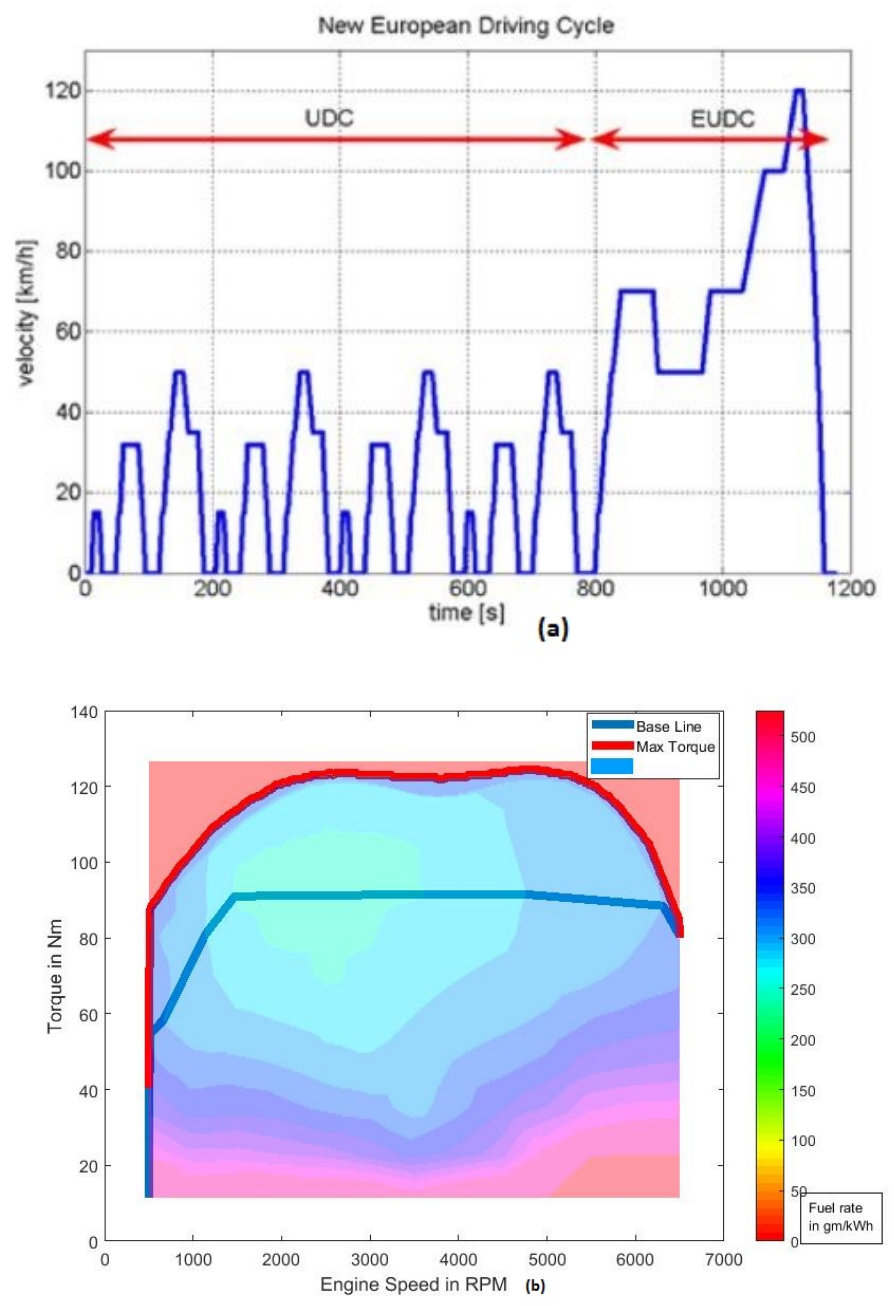

Fig. 1. (a) NEDC (b) BSFC map of 1.9L Saturn DOHC Engine

stochastically along with the slope of the road, which is detailed in this section.

1) Stochastic Drive Cycle Construction: In general, a driving cycle can be defined as a sequence of the following:

1. $t_{1}$ time to accelerate to speed $v_{2}$ from $v_{1}$

2. $t_{2}$ time to cruise at speed $v_{2}$

3. $t_{3}$ time to decelerate to a speed $v_{3}$

4. If the vehicle has not come to stop $\left(v_{3}>0\right)$, the vehicle cruise for another $t_{4}$ time at speed $v_{3}$

5. The next cycle starts at initial speed $v_{1}=v_{3}$ of the previous cycle

A city driving profile consists of following this sequence with different values of $t_{i} \mathrm{~s}$ and $v_{i} \mathrm{~s}$ repeatedly, where for most of the cases $v_{1}$ and $v_{3}$ being zero, representing a startstop scenario. Further, the slope of the road will also determine the traction force required for the vehicle. To implement a Monte Carlo simulation, input parameters need to be defined, along with their probability density function (PDF) [?]. The stochastic drive cycle is generated by the following method:

- An initial velocity $v_{1}$ is selected as same as the final velocity of the last drive cycle $\left(v_{3}\right)$, if it is more than 
$5 \mathrm{~km} / \mathrm{h}$, otherwise set to be 0 . For initialisation it is set to be 0

- A cruising velocity $v_{2}$ has been selected from a uniform PDF starting from $v_{1}+20 \mathrm{~km} / \mathrm{h}$ to $120 \mathrm{~km} / \mathrm{h}$

- Minimum time required to accelerate from $v_{1}$ to $v_{2}$ has been found to be $t_{1_{\text {min }}}$, using engine red-line Torquespeed curve

- Maximum time required to accelerate from $v_{1}$ to $v_{2}$ has been found to be $t_{1_{\max }}$, assuming maximum time required as twice of the same required for NEDC drive cycle

- Actual time required to accelerate from $v_{1}$ to $v_{2}$ has been selected from a normal distribution with mean $\mu$ at $\frac{t_{1_{\max }+t_{1_{\text {min }}}}}{2}$, and standard deviation (s.d) $\sigma$ at $\frac{t_{1_{\max }}-t_{1_{\min }}}{6}$, which ensures $6 \sigma$ coverage in between $t_{1_{\min }}$ and $t_{1_{\max }}$

- Cruising time $t_{2}$ has been selected from a uniform distribution in between $5 \mathrm{~s}$ to $60 \mathrm{~s}$.

- A final velocity $v_{3}$ is selected from an exponential probability distribution function (PDF), starting from 0 , up-to $v_{2}-20 \mathrm{~km} / \mathrm{h}$

- Min time to decelerate $t_{3_{\text {min }}}$ has been calculated using maximum declaration to be $2.5 \mathrm{Km} / \mathrm{h} / \mathrm{s}$

- Actual time to decelerate $t_{3}$ has been selected from a uniform distribution between $t_{3_{\text {min }}}$ and $2 t_{3_{\text {min }}}$

- If $v_{3}$ is more than $5 \mathrm{~km} / \mathrm{h}$ another cruising time $t_{4}$ has been selected from a uniform distribution in between $5 \mathrm{~s}$ to $30 \mathrm{~s}$.

- This ends a single drive cycle run. The next cycle starts from $v_{3}$ of the previous cycle with an acceleration. This loop-back construction allows a continuous driving pattern.

- Finally, a slope of the road has been selected from a normal distribution with mean at 0 and $\sigma$ to be 0.033 , allowing $6 \sigma$ coverage in between +0.1 to -0.1

\section{RESULTS}

Using the stochastically generated drive cycles, the torque and speed operating point, of the ISA based on the ISA operation algorithm is calculated at one second interval. Finally, the frequency of operation of each T-S region, (with 30RPM, and $5 \mathrm{Nm}$ wide segments in T-S plain) is calculated to find the loading pattern of the ISA. The SOC has been set to $50 \%$ at the start of the simulation, and updating at 1 second interval as per motor/generator operation. For this calculation, a flat $72 \%$ ISA efficiency has been assumed (80\% machine efficiency, $90 \%$ power electronic converter efficiency). Further, to emulate auxiliary loads, a constant 700w load has been applied to the battery. The simulation is run for 100,000 times resulting in more than $90,000 \mathrm{~km}$ distance and a duration of more than 2000 hour driving time. A sample drive pattern during the simulation is shown in Fig. 2 (a), along with the SOC of the battery in Fig. 2(b) for 0 - 1000 seconds.

The actual distribution of different parameters of the stochastic drive cycles used for the simulation is shown in Fig.

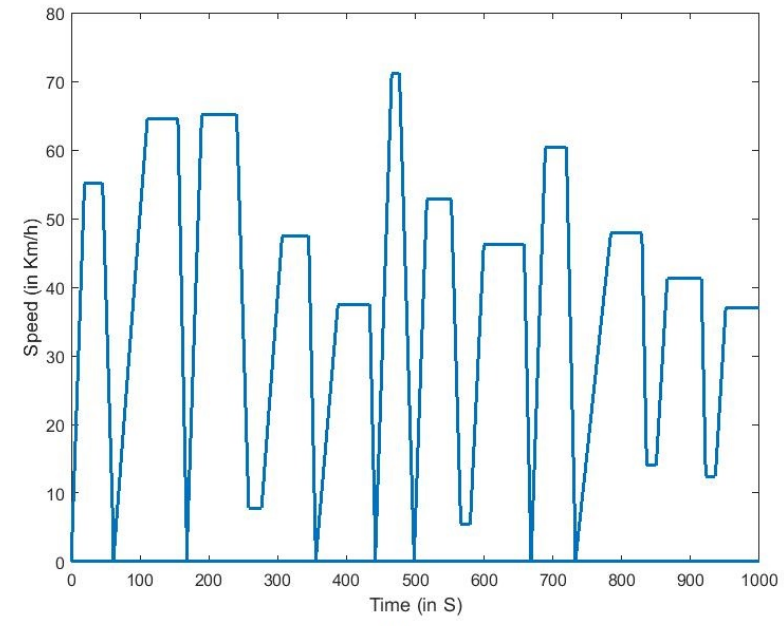

(a)

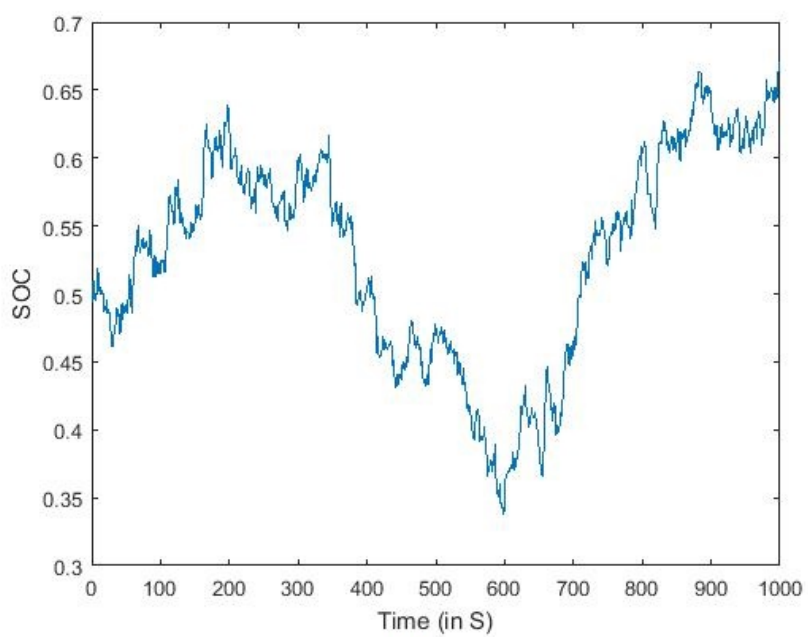

(b)

Fig. 2. (a) A sample drive pattern from the Monte Carlo Simulation (b) SOC during one of the drive cycle

3. It can be seen, the discussed probability density functions, in the previous section, has been clearly implemented.

Fig 4 shows the heat-map of the frequency of operation in both motoring and generating mode of the ISA. The colourmap in the figure shows the percentage of time each sector has been used over the net simulation time ( $>2000$ hour). Frequently used zones are also marked in the figure as well. The final T-S drive requirement characteristics, as shown in Fig. 4, has been obtained by covering the most frequently operated regions.

\section{Co-Simulation with ADVISOR}

Although the above-discussed methodology provides an initial estimation of the torque-speed operating region of the ISA machine, the detailed vehicle system is not modelled. This can be overcome by using quasi-static models of vehicle components, by modelling the power flow between them. 


\section{IEEE Transportation Electrification Conference (ITEC-India)}
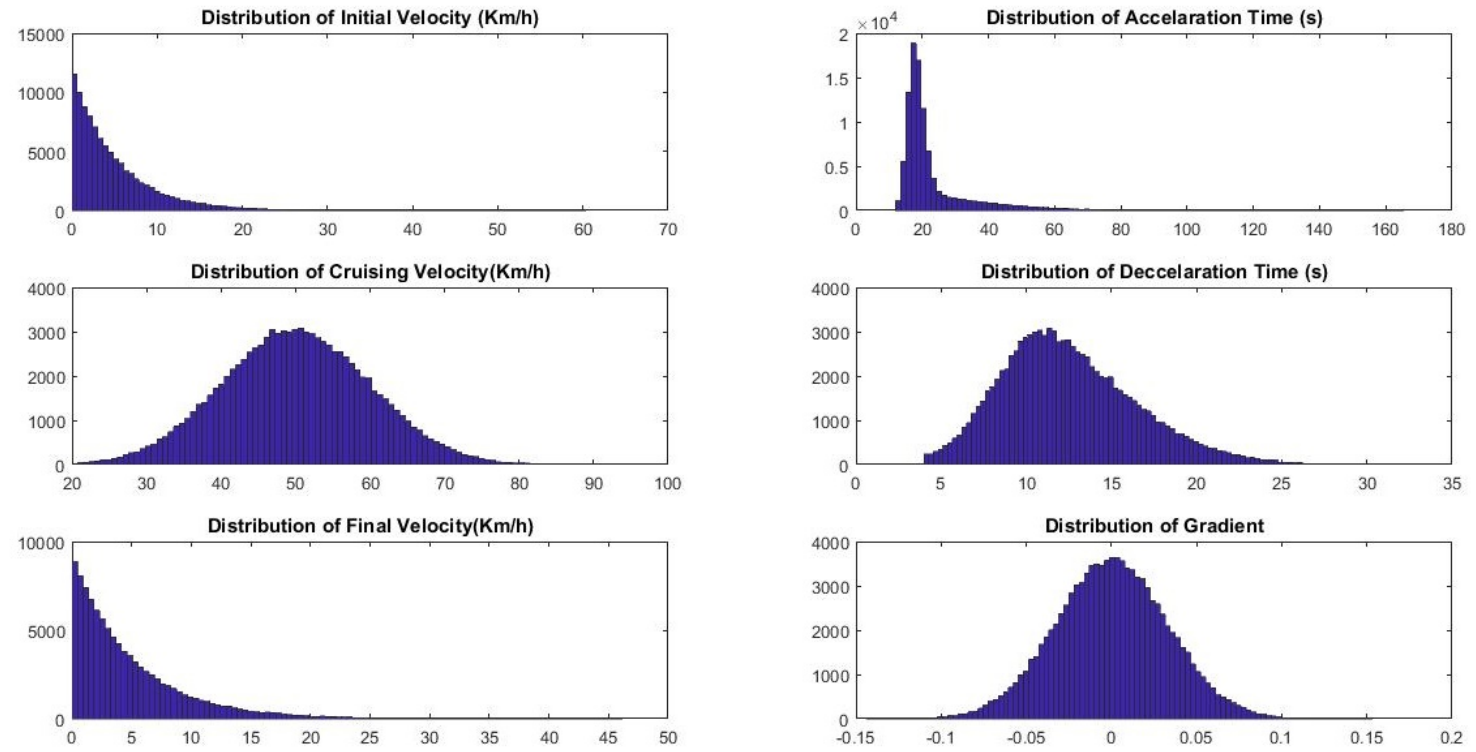

Fig. 3. Distribution of parameters of stochastic Drive Cycle under Monte Carlo Simulation

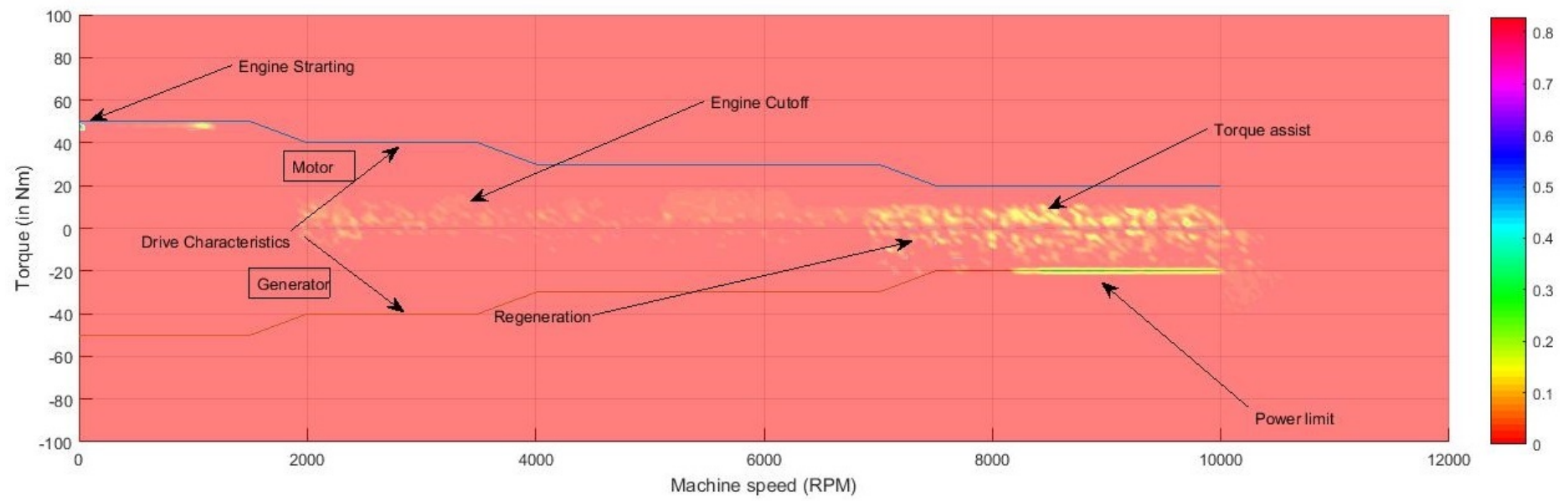

Fig. 4. Frequency of operation of ISA in T-S plain

ADVISOR [?], a MATLAB - SIMULINK based package, is often used for analysis of a designed power-train configuration under different test drive scenario and other tests such as acceleration test, gradient test. The package models the vehicle components, such as engine, gear box, battery, electric machine etc. dynamics for quasi-static simulation using steady state (e.g constant torque and speed), based on the test data. Here a complete parallel starter alternator vehicle model, using the default options in ADVISOR has been obtained. The model has been fed with stochastic drive cycles, generated from the reported Monte -Carlo simulation with 0.1s resolution. Due to the limitation of the quasi-static modelling approach of ADVISOR, the time resolution cannot be increased further. The inbuilt option of Fuzzy logic based power-train control strategy for the parallel starter alternator power train of ADVISOR has been used to control the vehicle The simulation results of the vehicle for each drive cycle is analysed, to find the torquespeed operating point of the starter-alternator machine. The detailed block diagram of the ADVISOR model is shown in Fig. 5. Similar to the analysis before, the simulation is run for 100,000 times resulting in more than 90,000 $\mathrm{km}$ distance and a duration of more than 2000 hour driving time. The obtained heat-map of the frequency of operation of the ISA in TorqueSpeed plain is shown in Fig. 6. It can be noted, due to optimal control strategy the operating region of ISA is now limited to lower speed range. It can be seen, similar to the result in the previous section, here also, there is a clear high torque, low-speed zone for engine starting, some intermittent medium speed-torque zone of operation torque assist, and a wide power limited regeneration zone. 


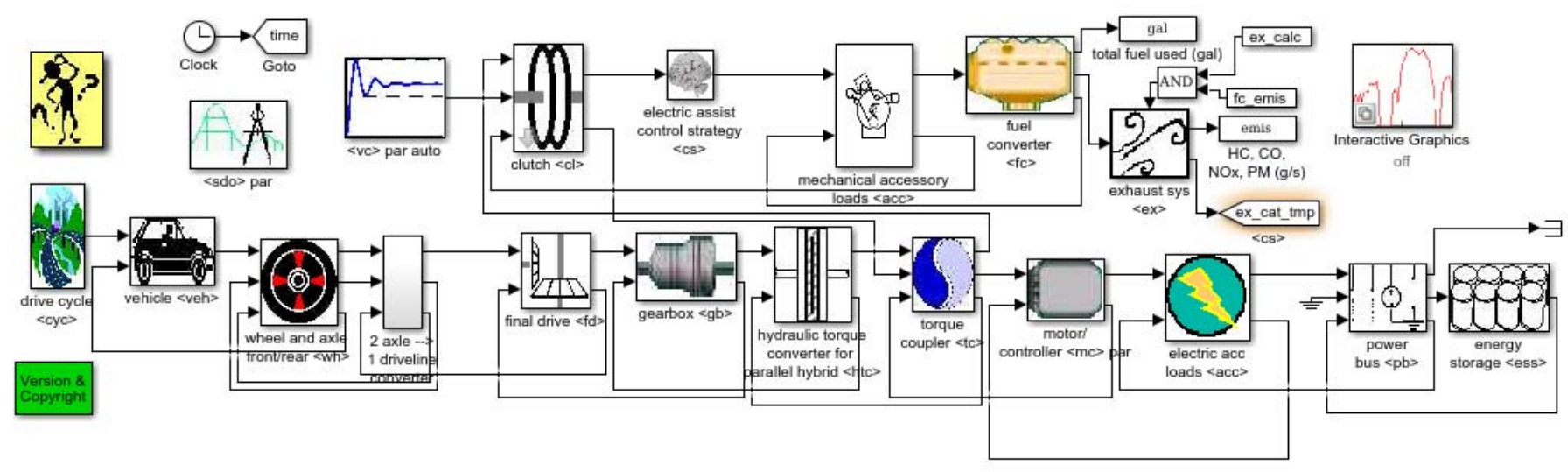

Fig. 5. Quasi Static Block diagram of vehicle model from ADVISOR

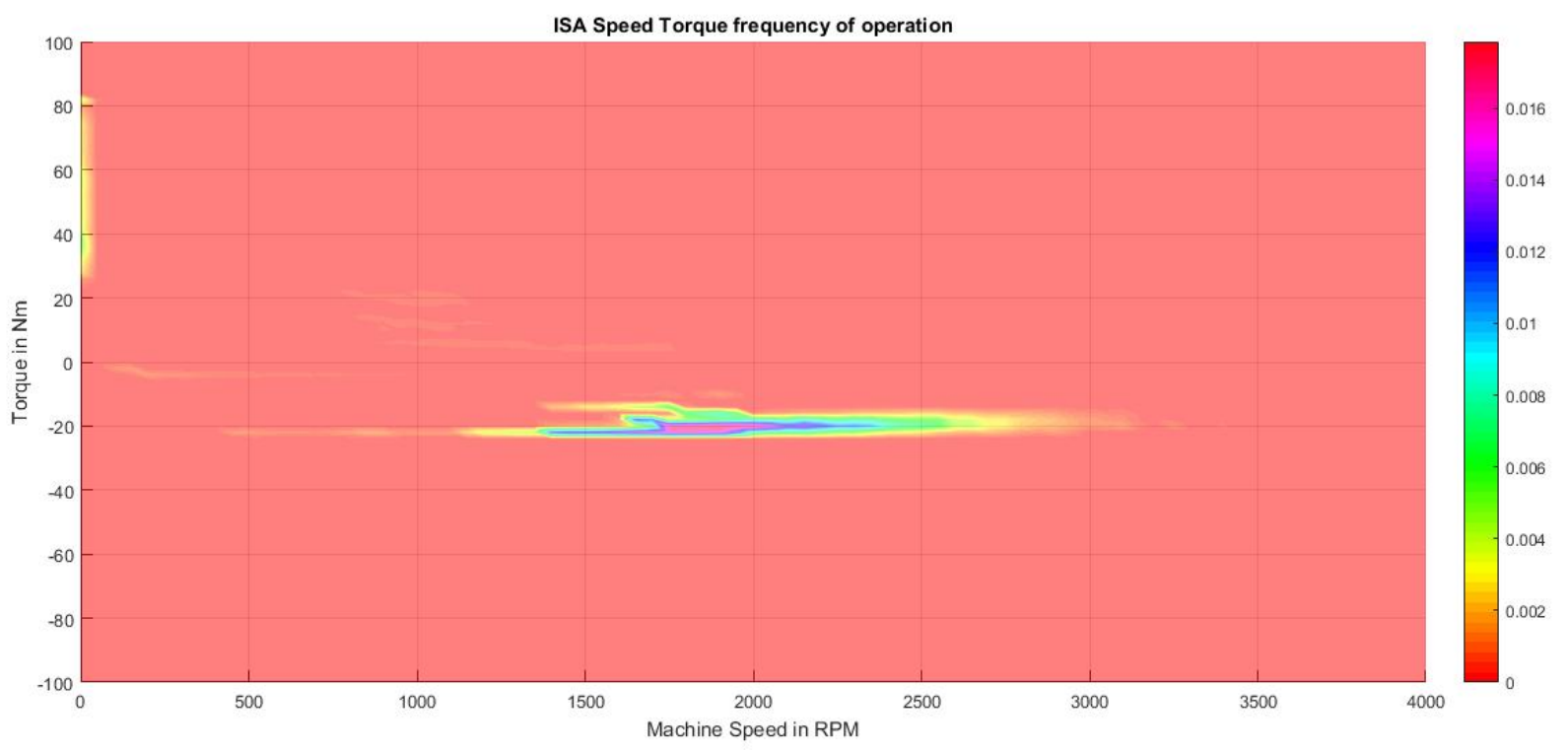

Fig. 6. Frequency of operation of ISA in T-S plain from ADVISOR - Co-Simulation

\section{CONCLUSions}

In this paper, a Monte Carlo simulation method to obtain the design requirements of an ISA has been reported. By constructing stochastic drive cycles, and using the ISA operation algorithm, the most frequent zones of operation of the machine in the T-S plane has been found This result has been used to obtain the required ISA T-S characteristics. Finally, in section 4, a co-simulation with ADVISOR has been reported to deploy the reported Monte-Carlo simulation, along with a quasi-static model of the vehicle for more accurate results. As the simulation covers a significant distance covered and driving time of the vehicle, the obtained drive characteristics, outlining frequent operating regions, can be taken as much more robust design characteristics for ISA design, compared to the standard drive cycle based design approach. Moreover, the methodology, of using Monte Carlo simulation can be used for any other automotive component, for which duty is dependent on the drive cycle of the vehicle. Furthermore, the generic approach can be utilised to develop the electric power train control algorithm, to ensure the component operating region covers the most common of the drive cycle scenarios. The simulation methodology can be particularly useful in the absence of driving test data, as obtaining driving profile data is often costly and time consuming. Further, in the presence of limited driving profile data, a simple analysis will enable the distribution PDFs of different parameters of the drive cycle used in this simulation to be obtained, which can be then used for a similar analysis. However, this simulation methodology allows detailed coverage of most frequently encountered scenarios, hence achieving the optimal ISA design. 


\section{IEEE Transportation Electrification Conference (ITEC-India)}

\section{ACKNOWLEDGEMENT}

The authors would like to acknowledge Commonwealth Scholarship Commission, UK and The Cambridge Commonwealth, European \& International Trust for their generous support to pursue this research.

\section{REFERENCES}

[1] H. Turker, "Methodology hybridization of a vehicle," in 2015 IEEE Transportation Electrification Conference and Expo (ITEC), pp. 1-8, June 2015.

[2] W. Cai, "Comparison and review of electric machines for integrated starter alternator applications," in Conference Record of the 2004 IEEE Industry Applications Conference, 2004. 39th IAS Annual Meeting, vol. 1, p. 393, Oct. 2004.

[3] Rotating electrical machines - Part 1: Rating and performance - IEC 60034-1:2017

[4] Faron, G., Pagerit, S., and Rousseau, A. (2009). Evaluation of PHEVs Fuel Efficiency and Cost Using Monte Carlo Analysis. Presented at the 24th International Electric Vehicle Symposium ( EVS24 ), Stavanger, Norway, May 2009.

[5] ADVISOR Advanced Vehicle Simulator ; web-link : http://adv-vehiclesim.sourceforge.net/ 